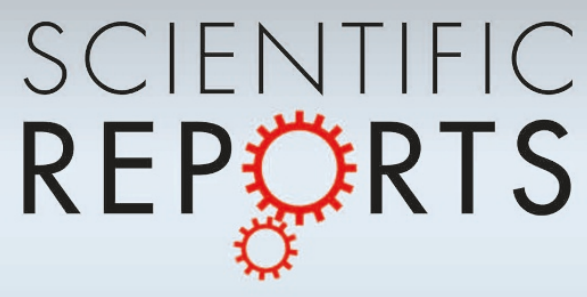

OPEN

SUBJECT AREAS:

FERROELECTRICS AND

MULTIFERROICS

ELECTRONIC PROPERTIES AND MATERIALS

Received

29 October 2014

Accepted

24 March 2015

Published

7 May 2015

Correspondence and requests for materials should be addressed to J.Z. (zhangiialiang@ sdu.edu.cn)

\section{Unfolding grain size effects in barium titanate ferroelectric ceramics}

\author{
Yongqiang Tan ${ }^{1,4}$, Jialiang Zhang', Yanqing Wu' , Chunlei Wang' ', Vladimir Koval ${ }^{2}$, Baogui Shi ${ }^{3}$, \\ Haitao $\mathrm{Ye}^{3}$, Ruth McKinnon ${ }^{4}$, Giuseppe Viola ${ }^{4,5}$ \& Haixue Yan $^{4}$
}

\begin{abstract}
${ }^{1}$ School of physics, State Key Laboratory of Crystal Materials, Shandong University, Jinan 250100, P. R. China, ${ }^{2}$ Institute of Materials Research, Slovak Academy of Sciences, Watsonova 47, 04001 Kosice, Slovakia, ${ }^{3}$ School of Engineering and Applied Science, Aston University, Birmingham B4 7ET, United Kingdom, ${ }^{4}$ School of Engineering and Materials Science, Queen Mary University of London, Mile End Road, London, E1 4NS, United Kingdom, ${ }^{5}$ Department of Applied Science and Technology, Institute of Materials Physics and Engineering, Corso Duca degli Abruzzi 24, 10129 Torino, Italy.
\end{abstract}

Grain size effects on the physical properties of polycrystalline ferroelectrics have been extensively studied for decades; however there are still major controversies regarding the dependence of the piezoelectric and ferroelectric properties on the grain size. Dense $\mathrm{BaTiO}_{3}$ ceramics with different grain sizes were fabricated by either conventional sintering or spark plasma sintering using micro- and nano-sized powders. The results show that the grain size effect on the dielectric permittivity is nearly independent of the sintering method and starting powder used. A peak in the permittivity is observed in all the ceramics with a grain size near $1 \mu \mathrm{m}$ and can be attributed to a maximum domain wall density and mobility. The piezoelectric coefficient $d_{33}$ and remnant polarization $P_{\mathrm{r}}$ show diverse grain size effects depending on the particle size of the starting powder and sintering temperature. This suggests that besides domain wall density, other factors such as back fields and point defects, which influence the domain wall mobility, could be responsible for the different grain size dependence observed in the dielectric and piezoelectric/ferroelectric properties. In cases where point defects are not the dominant contributor, the piezoelectric constant $d_{33}$ and the remnant polarization $P_{\mathrm{r}}$ increase with increasing grain size.

nderstanding the grain size effects that govern crystal structure and the functional properties of ferroelectrics is of vital importance in improving the performance of ferroelectric systems, which are embedded in a number of electronic devices, such as sensors, actuators, transducers and non-volatile memories ${ }^{1-3}$. Due to a growing demand for miniature devices, significant progress in the fabrication of micro-, meso- and nano-scale ferroelectric structures has been made ${ }^{4,5}$. A fundamental understanding of grain size effects on the dielectric and ferroelectric properties was achieved by studying low-dimensional ferroelectric structures ${ }^{6-12}$. Theoretical and experimental studies on thin/ultrathin film $\mathrm{s}^{6-8}$, nanowires ${ }^{9,10}$ and other types of nano-dimensional systems ${ }^{11,12}$ have shown that ferroelectricity persists down to the nanoscale, thereby demonstrating their potential for use in miniature devices. Nevertheless, certain applications require bulk components with specific functional properties, which can be directly obtained from a specific grain size. Although grain size effects on the dielectric, piezoelectric and ferroelectric properties have been widely studied in several ferroelectric bulk systems, there are still a number of aspects which remain unclear. These are mainly related to the grain size dependency of the piezoelectric and ferroelectric properties, often showing discrepancies in the existing literature. In addition, there are a number of other factors that could influence the grain size dependency; their identification is the main aim of the present study. Barium titanate ceramics are chosen as a model ferroelectric system for this research.

Barium titanate $\left(\mathrm{BaTiO}_{3}\right)$ is a typical ferroelectric material with a perovskite-type structure. It has been widely studied for dielectric capacitor and lead-free piezoelectric applications, achieving both high dielectric permittivity (up to 7000$)^{13}$ and piezoelectric constant $\left(d_{33}\right.$ up to $788 \mathrm{pC} / \mathrm{N}$ in textured ceramics) ${ }^{14}$ values. In $\mathrm{BaTiO}_{3}$ ceramics, the grain size has been reported to have substantial influence on the dielectric permittivity ${ }^{13,15-21}$. The dielectric constant of the $\mathrm{BaTiO}_{3}$ ceramics first increases with decreasing average grain size, reaching a maximum value in the $\sim 0.8-1.1 \mu \mathrm{m}$ grain size range, and then rapidly decreases with further decrease of grain size ${ }^{13,15-21}$. Similar behaviour has been observed in other ferroelectrics ${ }^{22-24}$. Generally, the grain size dependence of the dielectric permittivity shows consistent trends despite the use of different powder processing and sintering methods ${ }^{13,15-21}$. Regarding the physical origin of the maximum value of the permittivity, usually associated with an intermediate grain size of $\sim 1 \mu \mathrm{m}$, two alternate theories based on internal residual stress and domain wall motion have been developed over the past decades (see Ref. 19 for a review). Recent in situ high energy X-ray diffraction experiments 
performed on $\mathrm{BaTiO}_{3}$ ceramics with grain sizes in the range 0.32$3.5 \mu \mathrm{m}$ suggest that the maximum permittivity found around $1 \mu \mathrm{m}$ grain size is due to maximum domain wall contribution ${ }^{21}$.

On the other hand, the reported piezoelectric coefficient $d_{33}$ and its grain size dependence have shown contradicting results in the literature $^{14,21,25-32}$. For several decades, it has been accepted that the $\mathrm{BaTiO}_{3}$-based ceramics show only modest piezoelectric activity, with a piezoelectric constant $d_{33}$ lower than $190 \mathrm{pC} / \mathrm{N}^{25}$. Nevertheless, remarkably high $d_{33}$ values (up to $460 \mathrm{pC} / \mathrm{N}$ ) have been obtained over recent years for $\mathrm{BaTiO}_{3}$ ceramics (grain size around $1 \mu \mathrm{m}$ ) prepared from hydrothermally synthesized fine powders ${ }^{14,26-27}$. In addition, a peak of $338 \mathrm{pC} / \mathrm{N}$ in the piezoelectric constant was found around the grain size of $1 \mu \mathrm{m}$ in $\mathrm{BaTiO}_{3}$ ceramics prepared by solidstate reaction and conventional sintering ${ }^{28}$. Using conventional sintering, it is difficult to obtain dense $\mathrm{BaTiO}_{3}$ ceramics with an average grain size smaller than $1 \mu \mathrm{m}$, the relatively low density of these ceramics is considered to have caused the reduction of the piezoelectric constant when compared to the ceramics prepared from hydrothermal powders ${ }^{28}$. The $\mathrm{BaTiO}_{3}$ ceramics fabricated by the conventional solid state reaction route with a bimodal grain size distribution (large grains had an average size of about $7.0 \mu \mathrm{m}$ and small grains had an average size of about $0.8 \mu \mathrm{m}$ ) showed a high $d_{33}$ value of $419 \mathrm{pC} / \mathrm{N}$, which could not be solely attributed to a grain size effect ${ }^{29}$. Recently, a peak in the $d_{33}$ was found around $2 \mu \mathrm{m}$ grain size in barium titanate ceramics and it was concluded that like the permittivity, the maximization of the piezoelectric constant $d_{33}$ is due to movement of $90^{\circ}$ domain walls ${ }^{21}$. However, for $\mathrm{BaTiO}_{3}$ ceramics prepared from ultrafine powders with grain size ranging from 0.56 to $120 \mu \mathrm{m}$, a maximum $d_{33}$ value was reported at $8.9 \mu \mathrm{m}^{30}$, which is larger than the grain size reported in other studies $(1-2 \mu \mathrm{m})^{21}$. Additional discrepancies in the grain size dependence of the piezoelectric constant can be found in Refs. 31, 32.

These differences demonstrate that processing conditions can have a significant influence on the physical properties of ferroelectric ceramics. However, at present, the detailed mechanisms for the grain size dependence of the piezoelectric properties in the $\mathrm{BaTiO}_{3}$ ceramics prepared using different processing methods and different starting materials are still unclear.

To solve this controversy, the dependence of the dielectric, piezoelectric and ferroelectric properties on the average grain size of barium titanate ceramics prepared by conventional sintering (CS) and spark plasma sintering (SPS) using micro- and nano-sized powders (see Fig. S1 in supplementary figures) was systematically studied. SPS is an effective way to fabricate dense nanostructured ceramics $^{21,23,33}$, which could allow for the preparation of a series of fully dense $\mathrm{BaTiO}_{3}$ ceramics with a large range of grain sizes. Through this systematic comparison, the present study highlights the main factors responsible for the controversial grain size dependence previously reported. In addition, it also provides an advanced understanding of the underlying mechanisms of the grain size effect, which will have important implications in maximizing the dielectric, piezoelectric and ferroelectric/ferroelastic properties of other ferroelectric systems.

\section{Results}

Grain size dependence of permittivity and piezoelectric constant. Table 1 summarizes the room temperature dielectric and piezoelectric properties of poled $\mathrm{BaTiO}_{3}$ ceramics prepared by conventional sintering and SPS from the micro- and nano-sized $\mathrm{BaTiO}_{3}$ powders. In the table, the labels 'CS' and 'SPS' denote 'conventional sintering' and 'spark plasma sintering', respectively; the labels 'micro' and 'nano' describe ceramics sintered using microand nano-sized $\mathrm{BaTiO}_{3}$ powder, respectively; while the number after them indicates the sintering temperature. The values of the relative density, average grain size, piezoelectric constant $d_{33}$, orthorhombictetragonal phase transition temperature $T_{\mathrm{O}-\mathrm{T}}$ and Curie point $T_{\mathrm{C}}$ are also listed in the table. The $T_{\mathrm{O}-\mathrm{T}}$ and $T_{\mathrm{C}}$ values were determined from the peaks in the permittivity vs. temperature curves measured during heating. From Table 1 it can be seen that all $\mathrm{BaTiO}_{3}$ samples show high density. For CS-micro $\mathrm{BaTiO}_{3}$ ceramics, the relative density gradually increases from $95.2 \%$ to $98.6 \%$ and the average grain size increases from $1.3 \mu \mathrm{m}$ to $32.0 \mu \mathrm{m}$ with increasing sintering temperature. The relative density of all the SPS samples is larger than $98.5 \%$ and the average grain size increases from $0.6 \mu \mathrm{m}$ to $18.5 \mu \mathrm{m}$ with increasing sintering temperature.

The room temperature grain size dependence of the permittivity and piezoelectric constant of the poled $\mathrm{BaTiO}_{3}$ ceramics prepared by CS and SPS techniques are plotted using the data in Table 1 and the results are shown in Fig. 1a and Fig. 1b. The dielectric permittivity $\varepsilon$ ' shows a similar grain size dependence in ceramics sintered by the CS and SPS, as shown in Fig. 1a. The $\varepsilon$ ' value of the CS-micro ceramics increases from 2730 to 3220 with decreasing grain size (Fig. 1a). Analogously to CS, the permittivity of the SPS ceramics increases with the reduction of grain size and shows a peak around $1 \mu \mathrm{m}$. After further reduction of the grain size, the permittivity decreases (Fig. 1a). For SPS-micro $\mathrm{BaTiO}_{3}$ ceramics, the ceramic with an average grain size of $1.2 \mu \mathrm{m}$ shows the largest permittivity of 4450 . The permittivity of the SPS-nano ceramics shows a peak value of 5800 at around $1 \mu \mathrm{m}$ grain size (Fig. 1a). It can be seen that SPS $\mathrm{BaTiO}_{3}$ ceramics exhibit a larger permittivity than that of the CS-micro ceramics in the whole grain size range. Furthermore, for SPS ceramics, SPS-nano ceramics exhibit a larger permittivity than

Table 1 | Dielectric and piezoelectric properties of the poled $\mathrm{BaTiO}_{3}$ ceramics prepared from micro-sized and nano-sized powders at room temperature. The values of the relative density, grain size, piezoelectric constant $d_{33}$, orthorhombic-tetragonal transition temperature $T_{\text {O-T }}$ and Curie point $T_{\mathrm{C}}$ data are included

\begin{tabular}{|c|c|c|c|c|c|c|c|}
\hline Sintering Condition & Relative Density (\%) & Average Grain Size $(\mu \mathrm{m})$ & Permittivity at $1 \mathrm{kHz}$ & $\tan \delta(\%)$ & $d_{33}(p C / N)$ & $T_{\mathrm{C}}\left({ }^{\circ} \mathrm{C}\right)$ & $T_{\text {O-T }}\left({ }^{\circ} \mathrm{C}\right)$ \\
\hline CS-micro-1230 & 95.2 & 1.3 & 3220 & 2.30 & 410 & 120.2 & 28.1 \\
\hline CS-micro-1280 & 97.8 & 5.8 & 2970 & 2.17 & 300 & 121.5 & 25.7 \\
\hline CS-micro-1350 & 98.6 & 32.0 & 2730 & 2.44 & 180 & 122.3 & 23.2 \\
\hline SPS-micro- 1100 & 99.1 & 1.2 & 4450 & 2.60 & 339 & 121.2 & 30.2 \\
\hline SPS-micro-1120 & 99.0 & 1.9 & 3670 & 2.41 & 343 & 121.5 & 30.7 \\
\hline SPS-micro-1200 & 99.3 & 4.3 & 3400 & 2.35 & 432 & 123.6 & 26.5 \\
\hline SPS-nano-1020 & 98.5 & 0.9 & 5800 & 1.17 & 340 & 120.6 & 29.6 \\
\hline SPS-nano-1040 & 99.3 & 2.3 & 5080 & 1.19 & 375 & 123.8 & 24.9 \\
\hline SPS-nano-1060 & 99.2 & 4.5 & 4500 & 1.11 & 420 & 123.9 & 21.5 \\
\hline SPS-nano- 1160 & 99.5 & 9.6 & 3960 & 1.38 & 425 & 125.5 & 18.1 \\
\hline
\end{tabular}



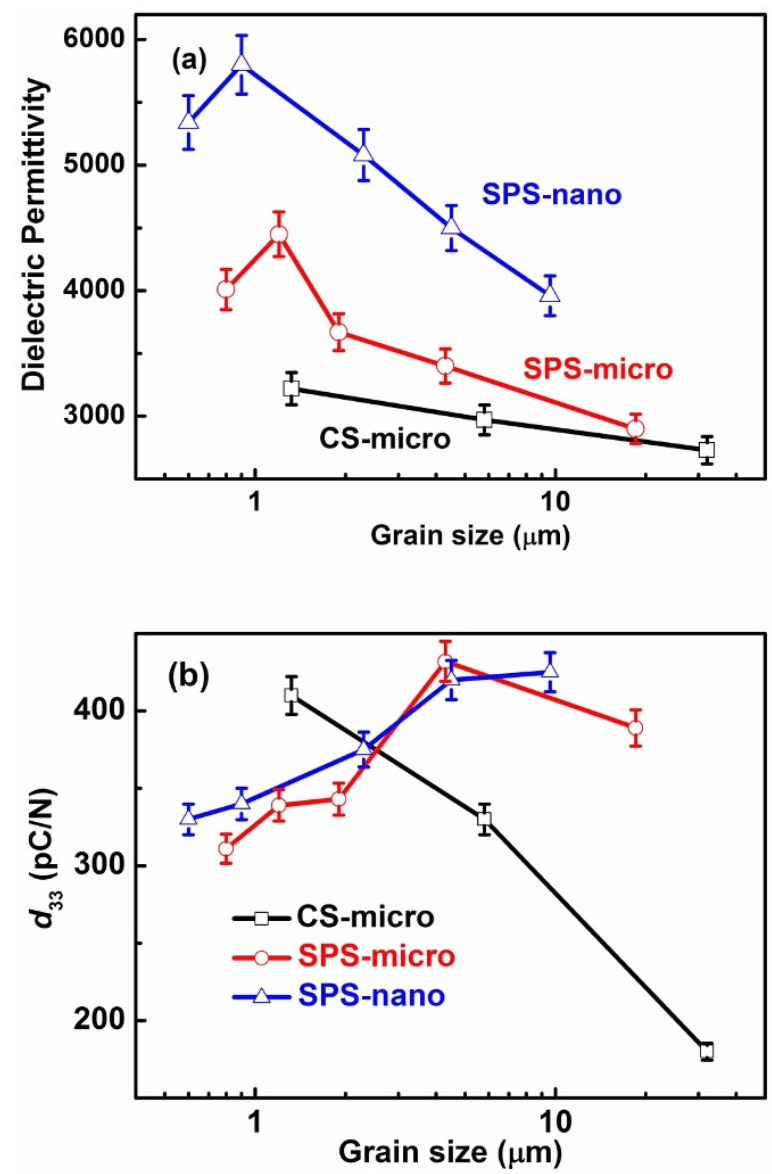

Figure 1 Grain size dependence of (a) the dielectric permittivity, and (b) the piezoelectric $d_{33}$ constant of the poled $\mathrm{BaTiO}_{3}$ ceramics prepared by the conventional and SPS techniques from micro- and nano-sized powders. The conventional sintering temperatures are $1230^{\circ} \mathrm{C}, 1280^{\circ} \mathrm{C}$ and $1350^{\circ} \mathrm{C}$; the SPS sintering temperatures for the micro-sized powder are $1080^{\circ} \mathrm{C}, 1100^{\circ} \mathrm{C}, 1120^{\circ} \mathrm{C}, 1200^{\circ} \mathrm{C}$ and $1240^{\circ} \mathrm{C}$, and for the nano-sized powder are $1000^{\circ} \mathrm{C}, 1020^{\circ} \mathrm{C}, 1040^{\circ} \mathrm{C}, 1060^{\circ} \mathrm{C}$ and $1160^{\circ} \mathrm{C}$.

SPS-micro ceramics in the whole grain size range (Fig. 1a). The grain size dependence of the permittivity observed in this study is in good agreement with the results reported in the literature, although the values show some variations due to the different raw powders and sintering techniques used ${ }^{13,15-21}$

The piezoelectric constant $d_{33}$ shows the opposite grain size dependence for the two different sintering methods (Fig. 1b). CSmicro $\mathrm{BaTiO}_{3}$ ceramics show a maximum $d_{33}$ value of $410 \mathrm{pC} / \mathrm{N}$ in CS-micro-1230 ceramic with an average grain size of $1.3 \mu \mathrm{m}$, which remarkably decreases with increasing grain size (Fig. 1b). The ceramic sintered at $1350^{\circ} \mathrm{C}$ shows a $d_{33}$ value of about $180 \mathrm{pC} / \mathrm{N}$ (Fig. 1b), which is consistent with the previous results for coarse grained $\mathrm{BaTiO}_{3}$ ceramics ${ }^{25,28}$. When using the SPS, the piezoelectric constant of the SPS-micro ceramics increases with increasing grain size and shows a maximum value of $430 \mathrm{pC} / \mathrm{N}$ in SPS-micro- 1200 ceramic with an average grain size of about $4 \mu \mathrm{m}$ (Fig. 1b). The $d_{33}$ value decreases with further increase of the sintering temperature above $1200^{\circ} \mathrm{C}$ (Fig. 1b). Owing to the fine particle size, the sintering temperatures of the SPS-nano ceramics are much lower than those of ceramics prepared from the micro-sized powder. All the SPS $\mathrm{BaTiO}_{3}$ ceramics prepared from the nano-sized powder show a high $d_{33}$ value $(>300 \mathrm{pC} / \mathrm{N})$. The maximum $d_{33}$ of SPS-nano $\mathrm{BaTiO}_{3}$ ceramics is close to the maximum $d_{33}$ of the SPS-micro ceramics. However, the $d_{33}$ coefficient of the SPS-nano ceramics, unlike that of the SPS-micro ceramics, increases with increasing grain size up to $9.6 \mu \mathrm{m}$. By comparing the piezoelectric constant of ceramics sintered by the two different methods, it can be inferred that there is a critical grain size of about $2 \mu \mathrm{m}$ below which CS-micro $\mathrm{BaTiO}_{3}$ ceramics show a larger $d_{33}$. The maximum $d_{33}$ for both the $\mathrm{BaTiO}_{3}$ ceramics sintered by the CS and SPS show a remarkably large value of over $400 \mathrm{pC} / \mathrm{N}$.

Grain size dependence of polarization-electric field hysteresis loops. Figure 2 shows the polarization-electric field (P-E) hysteresis loops of the unpoled $\mathrm{BaTiO}_{3}$ ceramics sintered by the CS and SPS. All of the ceramics except CS-micro-1350 show slim $\mathrm{P}-\mathrm{E}$ hysteresis loops with a coercive field $\left(E_{\mathrm{c}}\right)$ lower than $0.35 \mathrm{kV} /$ $\mathrm{mm}$, which is close to the value reported by other researchers ${ }^{25,31}$. The grain size dependence of the maximum polarization $\left(P_{\max }\right)$ and remnant polarization $\left(P_{\mathrm{r}}\right)$ of the CS and SPS $\mathrm{BaTiO}_{3}$ ceramics is shown in Fig. 3. It can be seen that for CS ceramics, both $P_{\max }$ and $P_{\mathrm{r}}$ decrease with increasing grain size (Figs. 3a, 3b). The CS-micro samples show a larger $P_{\mathrm{r}}$ than the SPS ceramics when the grain size is below $2 \mu \mathrm{m}$, and a lower value when the grain size is above $2 \mu \mathrm{m}$ (Fig. 3b). In the SPS-micro ceramics the maximum polarization $P_{\max }$ slightly increases with increasing grain size and the values are larger than those of the CS-micro samples. The remnant polarization $P_{\mathrm{r}}$ slightly increases and gradually saturates for grain sizes larger than $2 \mu \mathrm{m}$; meanwhile it dramatically decreases when the grain size is below $2 \mu \mathrm{m}$. For the SPS-nano ceramics, the $P_{\max }$ slightly increases with increasing grain size and $P_{\mathrm{r}}$ dramatically increases for grain sizes larger than $4.5 \mu \mathrm{m}$ (Fig. $3 \mathrm{~b}$ ), which is consistent with the grain size dependence of $d_{33}$ shown in Fig. 1. Figure 3c shows that the CSmicro-1350 sample (32 $\mu \mathrm{m}$ average grain size) exhibits a coercive field of $0.35 \mathrm{kV} / \mathrm{mm}$ which is larger than that of the CS-micro- 1230 $\left(1.3 \mu \mathrm{m}\right.$ average grain size; $\left.E_{\mathrm{c}}=0.17 \mathrm{kV} / \mathrm{mm}\right)$ and CS-micro- 1280 ceramics $\left(5.8 \mu \mathrm{m}\right.$ average grain size; $\left.E_{\mathrm{c}}=0.175 \mathrm{kV} / \mathrm{mm}\right)$. For the SPS-micro ceramics, $E_{\mathrm{c}}$ gradually decreases from $0.3 \mathrm{kV} / \mathrm{mm}$ to $0.17 \mathrm{kV} / \mathrm{mm}$ with increasing grain size. A similar grain size dependence of the coercive field is shown by the SPS-nano ceramics (Fig. 3c).

Grain size dependence of domain structure. Figure 4 shows SEM images of the domain patterns of several poled $\mathrm{BaTiO}_{3}$ ceramics prepared from micro- and nano-sized powders. The microstructures of the CS-micro ceramics are displayed in Figs. $4 \mathrm{a}$ and $4 \mathrm{~b}$. Figs. $4 \mathrm{c}$ to $4 \mathrm{f}$ show images of the SPS-micro ceramics, while Figs. $4 \mathrm{~g}$ and $4 \mathrm{~h}$ show the domain structures of two typical SPS-nano ceramics. For fine-grained $\mathrm{BaTiO}_{3}$ ceramics, the domain patterns mainly consist of stripes marked as ' $\mathrm{S}$ ' running across the whole grain, as visible in Figs. $4 \mathrm{a}$ and $4 \mathrm{c}$. The stripe lengths increase and the average domain width becomes larger as the grain size increases. The stripes are believed to correspond to $90^{\circ}$ domain patterns $s^{34,35}$. The formation of $90^{\circ}$ domains is a consequence of the relief of internal stresses in the $\mathrm{BaTiO}_{3}$ ceramics when cooled from a high temperature to below $T_{\mathrm{C}}{ }^{34,35}$. Herringbone patterns marked as ' $\mathrm{H}$ ' consisting of two adjacent sets of parallel stripes were occasionally found, especially in coarse grained samples with grain sizes larger than $2 \mu \mathrm{m}$, as shown in Figs. $4 \mathrm{~b}, 4 \mathrm{e}, 4 \mathrm{f}$ and $4 \mathrm{~h}$. These have been reported as typical domain structures of tetragonal $\mathrm{BaTiO}_{3}$ ceramics $^{34-38}$. In addition, a small amount of watermarks labelled as 'W' (believed to correspond to $180^{\circ}$-domain boundaries) ${ }^{28,34}$, were often observed in coarse grained $\mathrm{BaTiO}_{3}$ ceramics and rarely appear in fine grains (Figs. $4 \mathrm{~b}, 4 \mathrm{f}$ and $4 \mathrm{~h}$ ). This suggests the existence of $180^{\circ}$ domains in coarse grained $\mathrm{BaTiO}_{3}$ ceramics after poling, indicating that either it was not possible to align all the $180^{\circ}$ domains during poling or that the ceramics lost part of the domain alignment on removal of the field after the poling process.

The domain width of the striped domain structure was measured at a large number of locations in ceramics with different grain size and an average was calculated. The resultant grain size dependence of the average domain width for $\mathrm{BaTiO}_{3}$ ceramics prepared by different sintering methods is shown in Fig. 5. It can be seen that the average 

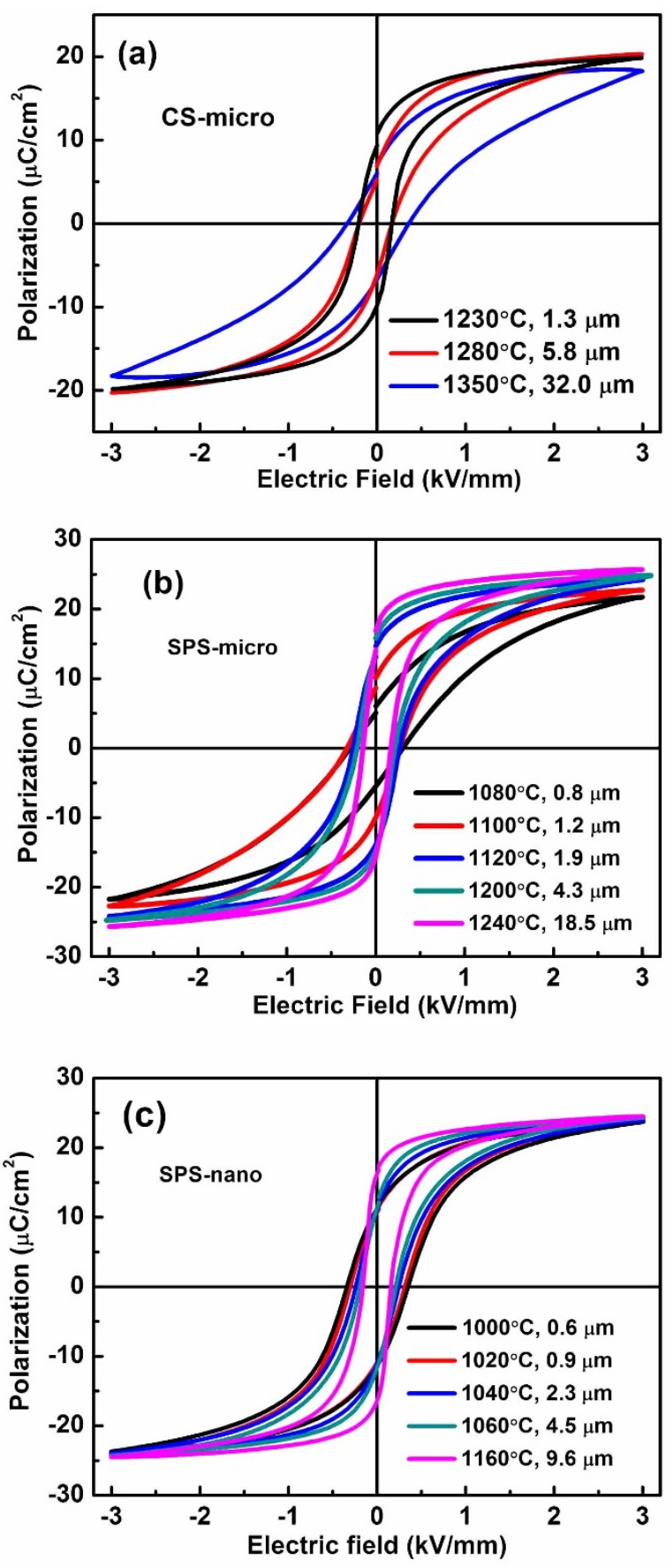

Figure 2 | P-E loops of $\mathrm{BaTiO}_{3}$ ceramics prepared from different powders and sintered at different temperatures. (a) CS-micro ceramics; (b) SPSmicro ceramics; (c) SPS-nano ceramics.

domain width decreases with decreasing grain size for all $\mathrm{BaTiO}_{3}$ ceramics. From Fig. 5 it is clear that there is a deviation from the parabolic relationship between domain width and grain size previously reported in ferroelectric ceramics ${ }^{36,39}$. Deviations from the parabolic law were also found in other ferroelectric systems and have been recently discussed in the literature ${ }^{40,41}$. When the grain size is larger than $4 \mu \mathrm{m}$, the CS ceramics and SPS ceramics show a similar grain size dependence of the domain width. The value of the domain width of the fine-grained $\mathrm{BaTiO}_{3}$ ceramics is consistent with that typically reported in literature $(\sim 100 \mathrm{~nm})^{17,28,29,31}$. The average domain width of the coarse-grained ceramics is much smaller than the previously reported values (larger than $500 \mathrm{~nm})^{17,34}$.
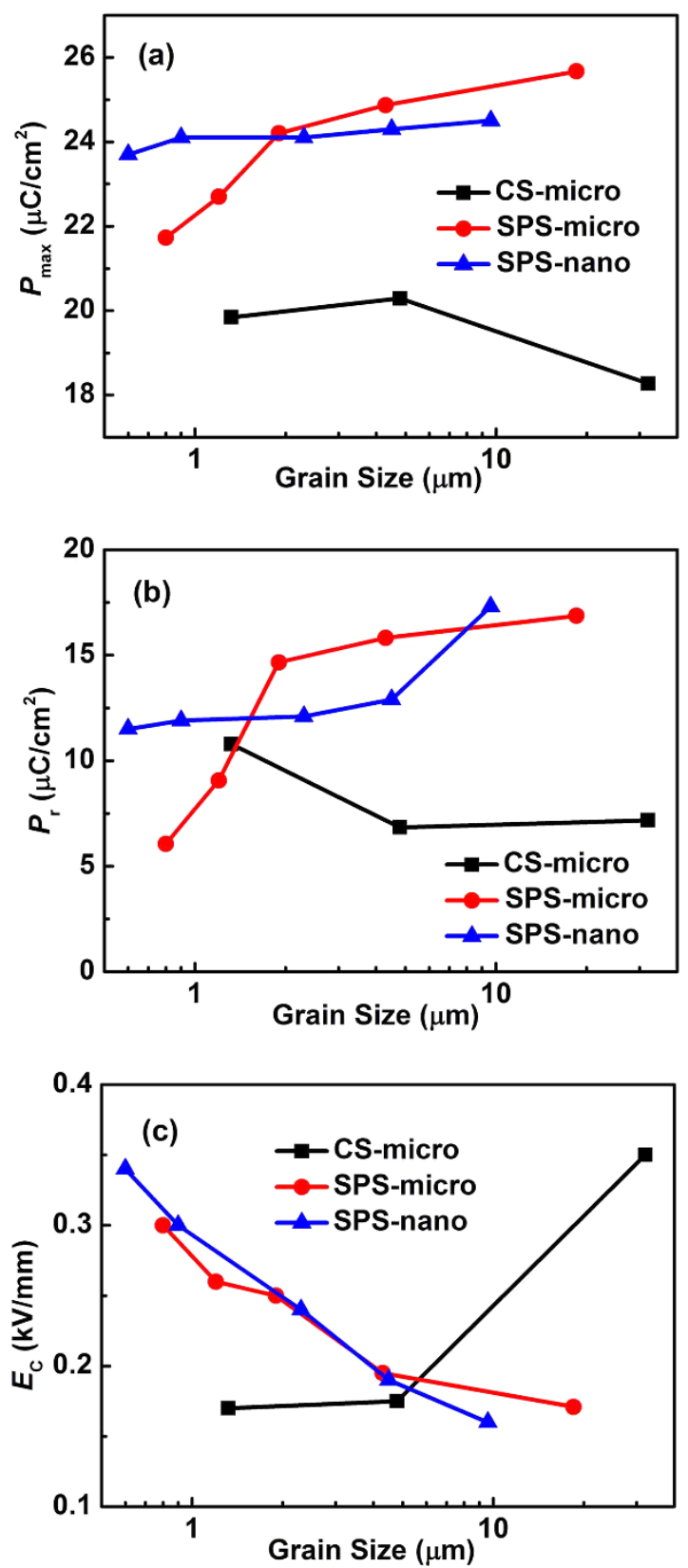

Figure $3 \mid$ Grain size dependence of (a) maximum polarization $P_{\max }$, (b) remnant polarization $P_{\mathrm{r}}$ and (c) coercive field $E_{\mathrm{C}}$ for $\mathrm{BaTiO}_{3}$ ceramics prepared by different sintering methods from micro- and nano-sized powders.

\section{Discussion}

It is well known that the dielectric and piezoelectric properties of ferroelectric ceramics include intrinsic and extrinsic contributions; the former originates from the deformation of the unit cell under an external electric or mechanical field, while the latter is mainly due to domain wall movement and point defects ${ }^{42-48}$. The domain wall contribution is determined by the domain wall density and domain wall mobility, which are also both influenced by many factors including grain size, back fields and defects ${ }^{42-48}$. The differences in the grain size dependence of the piezoelectric properties of the CS and SPS $\mathrm{BaTiO}_{3}$ ceramics can be interpreted based on the following aspects.

Domain wall density. The average domain width decreases with the reduction of the average grain size in $\mathrm{BaTiO}_{3}$ ceramics sintered by both methods, as demonstrated in Fig. 5, which is in agreement with 

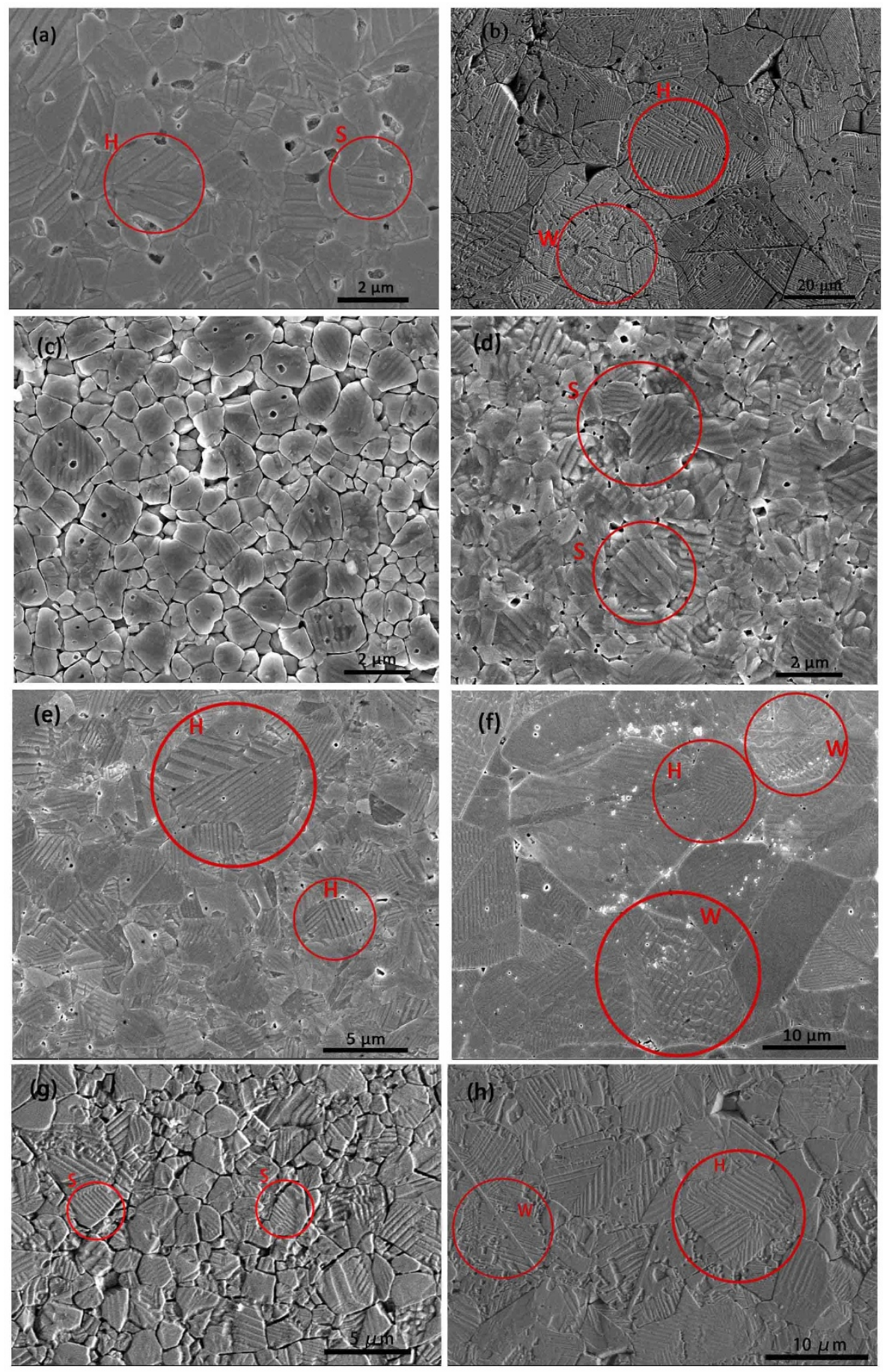

Figure $4 \mid$ Domain structures of $\mathrm{BaTiO}_{3}$ ceramics. (a) CS-micro-1230; (b) CS-micro-1350; (c) SPS-micro-1080; (d) SPS-micro-1120; (e) SPS-micro1200; (f) SPS-micro-1240; (g) SPS-nano-1040; (h) SPS-nano-1160. 'S' denotes stripe domain structure, 'H' denotes herringbone domain structure and 'W' denotes watermark domain structure.

Refs. 38, 40. This means that the number of domain walls per volume (the domain wall density) increases with decreasing grain size. This could contribute to a maximum in domain wall activity, which would produce an enhancement of the dielectric and piezoelectric properties in correspondence with a specific grain size. Optimum domain wall density could be one possible reason for the maximum permittivity observed near $1 \mu \mathrm{m}$ grain size in CS and SPS ceramics (see Fig. 1a), as also suggested in previous reports ${ }^{13,15-19,21}$. However, domain wall density is not the only factor which controls the domain wall contribution in ferroelectric/ferroelastic materials and additional factors should be taken into account in unravelling all the grain size effects observed.
Back fields. Back fields are a result of restoring forces acting on domain walls during domain switching ${ }^{49}$. The back fields may oppose the switching during electric field loading, and assist the back-switching during electric field unloading. A larger grain boundary area in fine grained ceramics would produce a back field which would exert a clamping effect on domain walls making the ferroelectric/ferroelastic domains harder to switch during the application of an electric field ${ }^{50,51}$. This explains why the $E_{c}$ value of the SPS ceramics decreases with increasing grain size (Fig. $3 c$ ). In the case of the SPS-nano ceramics, the piezoelectric constant and the remnant polarization both decrease with decreasing average grain size in the entire range studied (Figs. 1b, 3b). The decrease of $P_{\mathrm{r}}$ with 


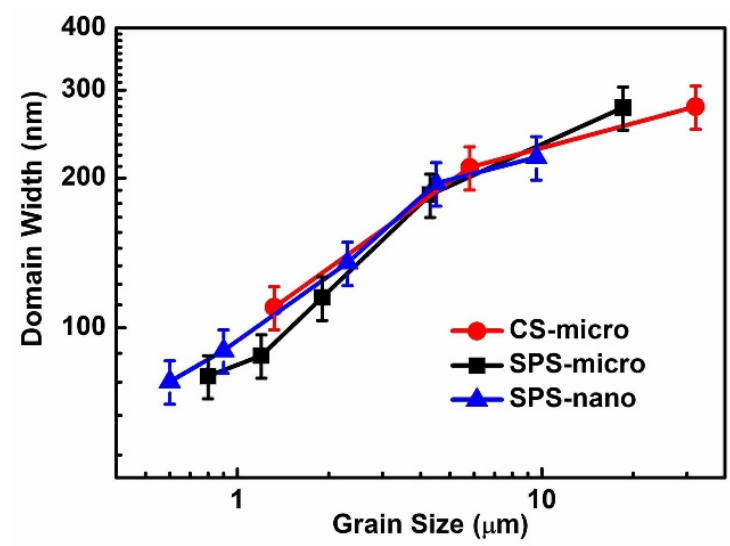

Figure 5 | Grain size dependence of the average domain width for the CS and $\mathrm{SPS} \mathrm{BaTiO}_{3}$ ceramics.

decreasing grain size can be attributed to an increased effect of the back field in ceramics with smaller grains. Back fields can also induce a reduction of the $d_{33}$ in accordance with the following scenarios, which may also overlap: a) the increased back field in ceramics with smaller grains might hinder domain alignments during DC poling; b) the back field reduces the alignment of the domains when the electric field is removed after the DC poling process.

Point defects. In order to achieve high density in conventional sintering, the sintering temperatures are higher and the dwelling times are much longer than those utilized in the SPS method. It was suggested that this represents a possible cause of point defects in the ceramics prepared by the conventional route ${ }^{52}$. Point defects tend to migrate to the domain boundaries or grain boundaries and subsequently pin the domain walls ${ }^{53-57}$. In our $\mathrm{BaTiO}_{3}$ samples, domain wall pinning effects seem to increase with increasing sintering temperature as demonstrated in Fig. 3 by the increase of $E_{\mathrm{c}}$ and the decrease of $P_{\max }$ with increasing grain size in CS-micro ceramics. Figure 6 shows the temperature dependence of the dielectric permittivity of the $\mathrm{BaTiO}_{3}$ ceramics prepared from different powders using different sintering methods. The peaks near $120^{\circ} \mathrm{C}$ correspond to the Curie point $T_{c}$ of $\mathrm{BaTiO}_{3}$. The broad peaks in the dielectric permittivity at high temperature from 400 to $700^{\circ} \mathrm{C}$, often observed in perovskite-type ferroelectric oxides including $\mathrm{BaTiO}_{3}$, can be attributed to the motion of oxygen vacancies as suggested by the calculated activation energy reported in Ref. 58. The peak intensity increases with increasing sintering temperature for CS-micro ceramics (Figs. 6a and b). In addition, the X-ray Photoelectron Spectroscopy (XPS) analysis shows that the valence state of barium and oxygen changes with increasing sintering temperature, reflecting possible modifications in the $\mathrm{Ba}$ $\mathrm{O}$ coordination and stoichiometry, which could be accommodated by the formation of oxygen vacancies (refer to supporting Figure S2). In Figs. $6 c$ and d, the intensity of the high temperature dielectric peaks decreases with reduction of the sintering temperature for SPS-micro ceramics. Further evidence for the presence of point defects (i.e. oxygen vacancies) in SPS-micro ceramics sintered at high temperatures are represented by the existence of an additional current peak that appears in the current-electric field (I-E) curves at temperatures starting from $80^{\circ} \mathrm{C}$ (refer to supporting Figures S3a and $\mathrm{S} 3 \mathrm{~b}$ ), an additional loss peak at a low frequency of about $100 \mathrm{~Hz}$ (refer to supporting Figures S3c and S3d) and by an asymmetric strain-electric field loop (refer to supporting Figures S3e and S3f). The high temperature broad peaks in the dielectric permittivity are almost absent in SPS-nano ceramics (Fig. 6e) due to the lower sintering temperature used.

The presence of point defects can reduce the domain wall mobility, thereby decreasing the extrinsic contribution of domain walls to the dielectric and piezoelectric properties. Domain wall pinning by point defects can have an important contribution to the reduction of the dielectric permittivity with increasing grain size in CS ceramics and for the lower permittivity of CS samples compared to SPS ceramics (Fig. 1a). For piezoelectric properties, the existence of point defects restricts domain switching under the DC poling process resulting in a poor domain alignment after poling, which leads to a lower piezoelectric constant. In addition, the small oscillating force used to measure the piezoelectric constant may not be large enough to counteract the pinning effect caused by the point defects resulting in the decrease of $d_{33}$ with increasing grain size in the CS-micro ceramics. In CS-micro ceramics, the remnant polarization and the piezoelectric constant both decrease with increasing grain size over the entire range studied. This suggests that the effect of domain wall pinning increases with increasing sintering temperature, resulting in a larger coercive field and a significant decrease of $P_{\max }$ in CS-micro ceramics with the largest grain size (see Figs. $1 b$ and $3 a-3 c$ ). In Fig. 2, the coarse grained SPS ceramics exhibit more saturated P-E hysteresis loops and much larger $P_{\mathrm{r}}$ values than those of the CS-micro ceramics. In addition, the maximum polarization $P_{\max }$ of SPS ceramics increases with increasing grain size in the range studied (Fig. 3a). The effects of sintering temperature on domain wall activity is also evidenced by the different grain size dependence of the piezoelectric constant observed for the SPS-micro ceramics and SPS-nano ceramics (Fig. 1). The latter were sintered at much lower temperatures and show a constant increase in the $d_{33}$ over the same grain size range. These observations indicate that when the sintering temperature becomes too high, the effect of domain wall pinning by point defects becomes the main factor in determining the observed grain size dependence of the piezoelectric and ferroelectric properties.

Origin of the different grain size dependencies of the permittivity and piezoelectric constant. In order to clarify the different grain size dependencies of the permittivity and piezoelectric constant, the SPSnano ceramics will be considered first as the contribution of point defects to the grain size dependence observed is presumably not dominant and the grain size dependence of the permittivity and $d_{33}$ shows the most remarkable differences among the ceramics studied here (Figs. 1a, 1b). Considering the P-E loop (Fig. 2c) and the S-P loop (Fig. S4c) of the SPS-nano-1160, it can be inferred that in the region around the coercive field the domain switching process is dominated by $180^{\circ}$ domain reorientation. This is evidenced by the steep change in polarization and the minor strain change in the region around the coercive field $(P=0)$, as shown in the hysteresis plots of Fig. $2 \mathrm{c}$ and Fig. S4c. In addition, the SEM images of the domain structure suggest an increased presence of $180^{\circ}$ domains in SPS-nano-1160 compared to the other SPS-nano ceramics. Therefore, it is unlikely that the permittivity of SPS-nano1160 is mainly dominated by the $180^{\circ}$ domain wall contribution; otherwise it would not be the lowest among all the SPS-nano ceramics (Fig. 1a). On the other hand, the $d_{33}$ of SPS-nano-1160 is the largest within the SPS-nano ceramics, due to the smaller back field experienced by this ceramic, which allowed for a higher degree of domain alignment during DC poling and a limited back-switching after poling. This is in agreement with the largest value of $P_{\mathrm{r}}$ in SPSnano-1160, among all the SPS-nano ceramics studied. Since it was deduced that the contribution of $180^{\circ}$ domain walls to the permittivity is not dominant, it can be inferred that the back field acts on $90^{\circ}$ domains. By definition, the dielectric permittivity indicates the amount of polarization change that can be induced under the application of an electric field, thus it should be expected that the permittivity generally decreases with increasing domain alignment after DC poling, as confirmed by previous experiments $^{28}$. Therefore the smallest permittivity in the SPSnano-1160 with the largest grain size among the poled SPS-nano ceramics should be attributed to a smaller contribution of $90^{\circ}$ 

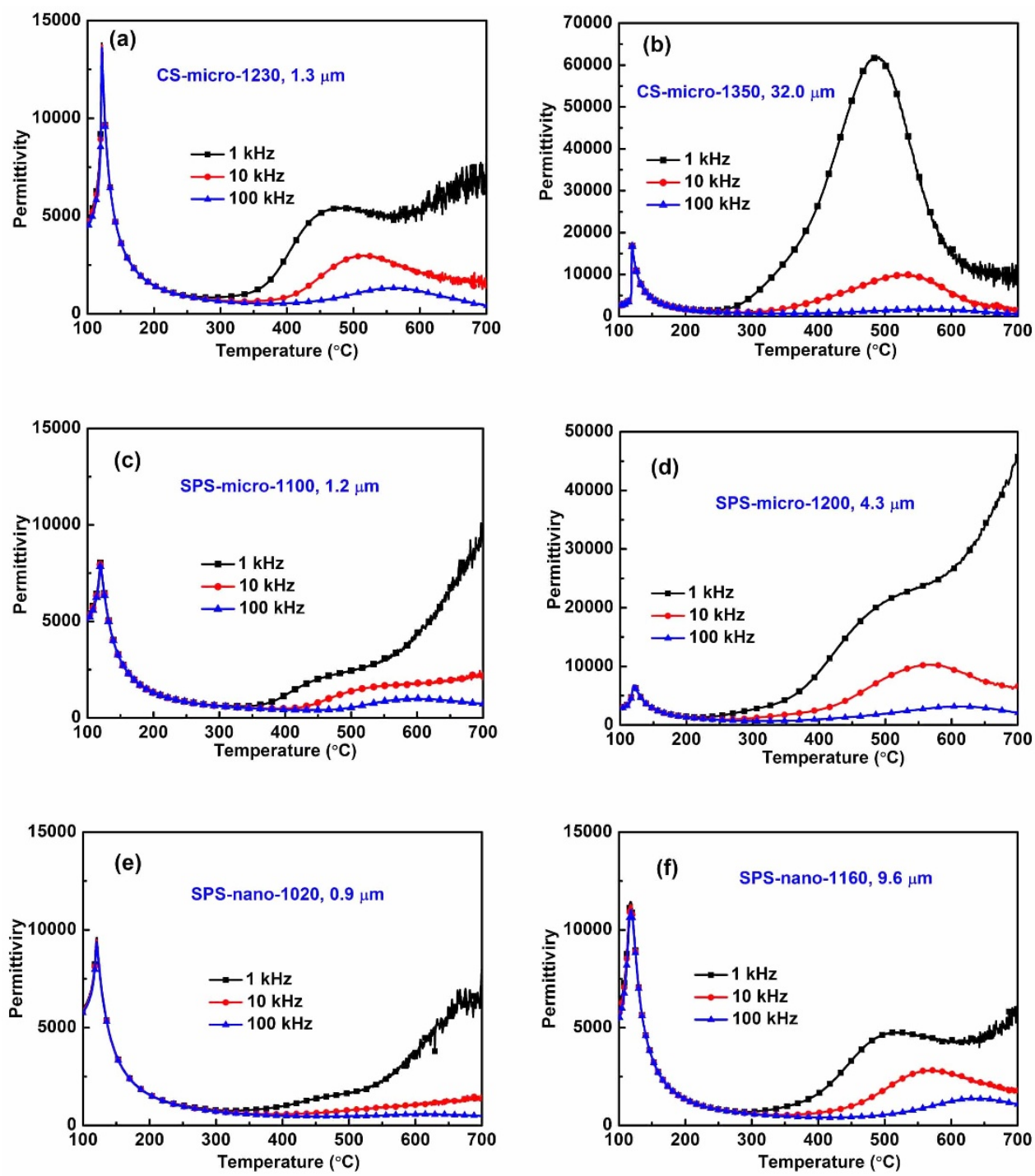

Figure 6 Temperature dependence of dielectric permittivity for $\mathrm{BaTiO}_{3}$ ceramics. (a), (b) CS-micro ceramics; (c), (d) SPS-micro ceramics; (e), (f) SPSnano ceramics.

domain walls due to a limited back-switching of $90^{\circ}$ domains after poling, in comparison to the other SPS-nano ceramics (see also Supporting Information S4). The dominant contribution of $90^{\circ}$ domain walls to the dielectric permittivity has been unambiguously proven in $\mathrm{PbZr}_{0.2} \mathrm{Ti}_{0.8} \mathrm{O}_{3}$ thin films ${ }^{59}$. With decreasing grain size, the $d_{33}$ decreases because of the increase of the back field according to the scenarios a) and b) previously described, while the permittivity increases, because of a larger contribution of $90^{\circ}$ domain walls. When the grain size becomes too small, the contribution of $90^{\circ}$ domain walls decreases and the permittivity of the poled ceramics starts to decrease with decreasing grain size (below $1 \mu \mathrm{m}$ in $\mathrm{BaTiO}_{3}$ ceramics). Similar arguments apply to the permittivity of the SPS-micro ceramics and the CSmicro ceramics, for which the grain size dependence of the $d_{33}$ has already been explained.

Summary of the observed grain size dependence. In the attempt to understand the grain size dependence observed in barium titanate ferroelectric ceramics, highly convoluted effects were observed on dielectric, piezoelectric and ferroelectric properties, which can be summarized as follows. The grain size dependence of the dielectric permittivity is mostly independent of both the starting powders and sintering process used. The maximum dielectric permittivity at a critical grain size of about $1 \mu \mathrm{m}$ is mainly achieved by optimum density and mobility of $90^{\circ}$ domain walls.

The grain size dependence of the piezoelectric constant is instead affected by additional factors related to ceramic processing, including particle size of the starting powder and sintering temperature. With increasing grain size, the $d_{33}$ of CS-micro ceramics decreases over the whole grain size range; the $d_{33}$ of SPS-micro ceramics first increases and then decreases with a peak at $4.3 \mu \mathrm{m}$ grain size; the $d_{33}$ of SPSnano ceramics increases over the entire grain size range studied. The increase of $d_{33}$ in the SPS-nano ceramics and in the SPS-micro ceramics below $4.3 \mu \mathrm{m}$ is due to an increased domain alignment caused by a reduction of the back field exerted by grain boundaries with increasing grain size. Domain wall pinning by point defects is instead the main factor for the decrease of the $d_{33}$ in SPS-micro ceramics above $4.3 \mu \mathrm{m}$ and in CS-micro ceramics over the entire grain size range.

Regarding the ferroelectric properties, it was observed that the coercive field of SPS ceramics decreases with increasing grain size, while in CS ceramics it increases in ceramics with larger grains. The latter is attributed to an increased pinning effect on domain walls by point defects developed in ceramics sintered at high temperature. In ceramics where the point defects contribution is not dominant, the maximum and remnant polarization increase with increasing grain size. 


\section{Conclusions}

Grain size effects on dielectric, piezoelectric and ferroelectric properties of three different groups of $\mathrm{BaTiO}_{3}$ ceramics with distinct sintering temperature ranges were studied. This allowed for a wider view of the possible grain size dependencies which could be observed in ferroelectric ceramics. Grain size effect is influenced by contributions from ferroelectric domain walls, back fields and point defects developed during sintering at high temperatures. The maximum dielectric permittivity near $1 \mu \mathrm{m}$ grain size is achieved by optimum density and mobility of $90^{\circ}$ domain walls in all the $\mathrm{BaTiO}_{3}$ ceramics studied. In ceramics sintered by SPS at low temperatures $(T<$ $1200^{\circ} \mathrm{C}$ ), the grain size dependence of the piezoelectric and ferroelectric properties can be mainly attributed to the degree of domain alignment and the influence of the back field exerted by the grain boundaries. In ceramics sintered at high temperatures either by SPS or CS, there is an additional contribution from point defects, which increases with increasing sintering temperature and influences the domain wall contribution to the grain size effect observed. When studying grain size effects in ferroelectric materials, the point defects contribution should be minimized to avoid contradicting and misleading observations.

\section{Methods}

Powder preparation. Conventional micro-sized $\mathrm{BaTiO}_{3}$ powders were prepared from commercial $\mathrm{BaCO}_{3}$ (purity $\geq 99.0 \%$ ) and $\mathrm{TiO}_{2}$ (purity $\geq 99.8 \%$ ) powders. The $\mathrm{BaCO}_{3}$ and $\mathrm{TiO}_{2}$ powders were weighed according to the stoichiometric formula and ball-milled for $12 \mathrm{~h}$ in nylon pots with $\mathrm{ZrO}_{2}$ balls and alcohol as a milling media. The slurry was then dried and ground using an agate mortar and pestle. The powder mixture was calcined at $1050^{\circ} \mathrm{C}$ for $4 \mathrm{~h}$. After the second ball-milling, a fine $\mathrm{BaTiO}_{3}$ powder with average particle size lower than $0.5 \mu \mathrm{m}$ was obtained. Hydrothermal synthesized nano-powders of $\mathrm{BaTiO}_{3}$ with a particle size of $100 \mathrm{~nm}$ were also used as the precursor materials. The microstructures of different $\mathrm{BaTiO}_{3}$ powders are shown in Fig. S1.

Sintering. Two kinds of sintering techniques were adopted to sinter $\mathrm{BaTiO}_{3}$ ceramics: conventional sintering (CS) and Spark Plasma Sintering (SPS). In the case of CS, the micro-sized $\mathrm{BaTiO}_{3}$ powders (Fig. S1a) were pressed into pellets of $15 \mathrm{~mm}$ diameter and $1 \mathrm{~mm}$ thickness, and then sintered at $1210^{\circ} \mathrm{C}, 1280^{\circ} \mathrm{C}$ and $1350^{\circ} \mathrm{C}$ for $2 \mathrm{~h}$ in air (referred as CS-micro-1210, CS-micro-1280, and CS-micro-1350 for abbreviation). In the case of the SPS samples, $\mathrm{BaTiO}_{3}$ micro-sized powders were sintered in vacuum for 5 minutes under an uniaxial pressure of $85 \mathrm{MPa}$ at $1080^{\circ} \mathrm{C}, 1100^{\circ} \mathrm{C}, 1120^{\circ} \mathrm{C}$, $1200^{\circ} \mathrm{C}$ and $1240^{\circ} \mathrm{C}$ (referred as SPS-micro-1080, SPS-micro-1100, SPS-micro-1120, SPS-micro-1200 and SPS-micro-1240 for abbreviation) using a SPS furnace (HPD25/1 FCT systeme $\mathrm{GmbH}$ ). The $100 \mathrm{~nm}$ nanopowder (Fig. S1b) was sintered by the SPS unit at $1000^{\circ} \mathrm{C}, 1020^{\circ} \mathrm{C}, 1040^{\circ} \mathrm{C}, 1060^{\circ} \mathrm{C}$ and $1160^{\circ} \mathrm{C}$ (referred as SPS-nano-1000, SPS-nano-1020, SPS-nano-1040, SPS-nano-1060 and SPS-nano-1160 for abbreviation). All the SPS samples were annealed in air at $900^{\circ} \mathrm{C}$ for $2 \mathrm{~h}$ to eliminate the presence of any residual carbon and reduction effects incurred during SPS process.

Characterization. The density of the ceramics was measured by the Archimedes method. For electrical characterization, disk-shaped specimens were coated with a silver paint (Gwent Electronic Materials Ltd, C2011004D5, Pontypool, UK) on the top and bottom surfaces and fired at $600^{\circ} \mathrm{C}$ for $20 \mathrm{~min}$. Poling was carried out at $105^{\circ} \mathrm{C}$ in silicon oil under an electric field of $3.0 \mathrm{kV} / \mathrm{mm}$ for $30 \mathrm{~min}$. The piezoelectric $d_{33}$ coefficient was measured using a Berlincourt-type $d_{33}$ meter (model YE 2730A, Sinocera Piezotronics, China). The dielectric properties were measured using an impedance analyzer (Agilent 4294A). The polarization hysteresis (P-E) loops were traced using a ferroelectric hysteresis measurement tester (NPL, Teddingdon, $\mathrm{UK})^{60,61}$. For the microstructure and domain configuration characterization, the poled specimens were mirror-polished and chemically etched for $10 \mathrm{~s}$ in an aqueous solution of $5 \% \mathrm{HCl}$ with a small amount of $\mathrm{HF}$ ( 3 drops of $\mathrm{HF}: 20 \mathrm{ml} \mathrm{HCl}$ solution). The microstructure and domain structure observations were performed using a scanning electron microscope (SEM; model JEOL JSM 6300). X-ray photoelectron spectroscopy (XPS; ESCALAB MK II, VG Scientific) was carried out to study the valence state of ions in the ceramics sintered at different temperatures.

1. Haertling, G. H. Ferroelectric ceramics: history and technology. J. Am. Ceram. Soc. 82, 797-818 (1999)

2. Scott, J. F. Applications of modern ferroelectrics. Science 315, 954-957 (2007).

3. Yang, L. F. et al. Bipolar loop-like non-volatile strain in the (001)-oriented $\mathrm{Pb}$ $\left(\mathrm{Mg}_{1 / 3} \mathrm{Nb}_{2 / 3}\right) \mathrm{O}_{3}-\mathrm{PbTiO}_{3}$ single crystals. Sci. Rep. 4, 4591 (2014).

4. Dawber, M. K., Rabe, M. \& Scott, J. F. Physics of thin-film ferroelectric oxides. Rev. Mod. Phys. 77, 1083-1130 (2005).
5. Gruverman, A. \& Kholkin, A. Nanoscale ferroelectrics: processing, characterization and future trends. Rep. Prog. Phys. 69, 2443-2474 (2006).

6. Shaw, T. M., Trolier-McKinstry, S. \& McIntyre, P. C. The Properties of ferroelectric films at small dimensions. Annu. Rev. Mater. Sci. 30, 263-298 (2000).

7. Fong, D. D. et al. Ferroelectricity in ultrathin perovskite films. Science 304, 1650-1653 (2004).

8. Spaldin, N. A. Fundamental size limits in ferroelectricity. Science 304, 1606-1607 (2004).

9. Spanier, J. E. et al. Ferroelectric phase transition in individual single-crystalline $\mathrm{BaTiO}_{3}$ nanowires. Nano Lett. 6, 735-739 (2006).

10. Yun, W. S., Urban, J. J., Gu, Q. \& Park, H. K. Ferroelectric properties of individual barium titanate nanowires investigated by scanned probe microscopy. Nano Lett. 2, 447-450 (2002).

11. Polking, M. J. et al. Ferroelectric order in individual nanometre-scale crystals. Nat. Mater. 11, 700-709 (2012).

12. Wu, C. M. et al. Controllability of vortex domain structure in ferroelectric nanodot: fruitful domain patterns and transformation paths. Sci. Rep. 4, 3946 (2014).

13. Hoshina, T. et al. Domain size effect on dielectric properties of barium titanate ceramics. Jpn. J. Appl. Phys. 47, 7607-7611 (2008).

14. Wada, S. et al. Preparation of [110] grain oriented barium titanate ceramics by templated grain growth method and their piezoelectric properties. Jpn. J. Appl. Phys. 46, 7039-7043 (2007).

15. Kniepkamp, H. \& Heywang, W. Z. Depolarisationseffekte in polykristallin gesinterem $\mathrm{BaTiO}_{3}$. Angew. Phys. 6, 385 (1954).

16. Kinoshita, K. \& Yamaji, A. Grain-size effects on dielectric properties in barium titanate ceramics. J. Appl. Phys. 47, 371-373 (1976).

17. Arlt, G., Hennings, D. \& De With, G. Dielectric properties of fine-grained barium-titanate ceramics. J. Appl. Phys. 58, 1619-1625 (1985).

18. Bell, A. J., Moulson, A. J. \& Cross, L. E. The effect of grain size on the permittivity of $\mathrm{BaTiO}_{3}$. Ferroelectrics 54, 147-150 (1984).

19. Bell, A. J. Grain size effects in barium titanate - revisited. Paper presented at Applications of Ferroelectrics, Proceedings of the Ninth IEEE International Symposium, University Park, PA. IEEE. (DOI:10.1109/ISAF.1994.522286) (7 Aug 1991).

20. Buessem, W. R., Cross, L. E. \& Goswami, A. K. Phenomenological theory of high permittivity in fine-grained barium titanate. J. Am. Ceram. Soc. 49, 33-36 (1966).

21. Ghosh, D. et al. Domain wall displacement is the origin of superior permittivity and piezoelectricity in $\mathrm{BaTiO}_{3}$ at intermediate grain sizes. Adv. Func. Mater. 24, 885-896 (2014)

22. Randall, C. A., Kim, N., Kucera, J., Cao, W. W. \& Shrout, T. R. Intrinsic and extrinsic size effects in fine-grained morphotropic-phase-boundary lead zirconate titanate ceramics. J. Am. Ceram. Soc. 81, 677-688 (1988).

23. Zhang, H. T. et al. The grain size effect on the properties of Aurivillius phase $\mathrm{Bi}_{3.15} \mathrm{Nd}_{0.85} \mathrm{Ti}_{3} \mathrm{O}_{12}$ ferroelectric ceramics. Nanotechnology 20, 385708 (2009).

24. Huo, S. X., Yuan, S. L., Tian, Z. M., Wang, C. H. \& Qiu, Y. Grain size effects on the ferroelectric and piezoelectric properties of $\mathrm{Na}_{0.5} \mathrm{~K}_{0.5} \mathrm{NbO}_{3}$ ceramics prepared by pechini method. J. Am. Ceram. Soc. 95, 1383-1387 (2012).

25. Jaffe, B., Cook, W. R. \& Jaffe, H. Piezoelectric Ceramics. London: Academic, UK, 1971.

26. Takahashi, H. et al. Lead-free barium titanate ceramics with large piezoelectric constant fabricated by microwave sintering. Jpn. J. Appl. Phys. 45, L30-L32 (2006).

27. Karaki, T., Yan, K. \& Adachi, M. Barium titanate piezoelectric ceramics manufactured by two-step sintering. Jpn. J. Appl. Phys., 46, 7035-7038 (2007).

28. Zheng, P., Zhang, J. L., Tan, Y. Q. \& Wang, C. L. Grain-size effects on dielectric and piezoelectric properties of poled $\mathrm{BaTiO}_{3}$ ceramics. Acta Mater. 60, 5022-5030 (2012).

29. Shao, S. F. et al. High piezoelectric properties and domain configuration in $\mathrm{BaTiO}_{3}$ ceramics obtained through solid-state reaction route. J. Physics D: Appl. Phys., 41, 125408 (2008).

30. Ding, S. H., Song, T. X., Yang, X. J. \& Luo, G. H. Effect of grain size of $\mathrm{BaTiO}_{3}$ ceramics on dielectric properties. Ferroelectrics 402, 55-59 (2010).

31. Huan, Y., Wang, X. H., Fang, J. \& Li, L. T. Grain size effects on piezoelectric properties and domain structure of $\mathrm{BaTiO}_{3}$ ceramics prepared by two-step sintering. J. Am. Ceram. Soc. 96, 3369-3371 (2013).

32. Egerton, L. \& Koonce, S. E. Effect of firing cycle on structure and some dielectric and piezoelectric properties of barium titanate ceramic. J. Am. Ceram. Soc. 38, 412-418 (1955).

33. Viola, G. et al. Effect of grain size on domain structures, dielectric and thermal depoling of Nd-substituted bismuth titanate ceramics. Appl. Phys. Lett. 103, 182903 (2013).

34. Arlt, G. \& Sasko, P. Domain configuration and equilibrium size of domains in $\mathrm{BaTiO}_{3}$ ceramics. J. Appl. Phys. 51, 4956-4960 (1980).

35. Arlt, G. Twinning in ferroelectric and ferroelastic ceramics: stress relief. J. Mater Sci. 25, 2655-2666 (1990).

36. Baxter, P., Hellicar, N. J. \& Lewis, B. Effect of additives of limited solid solubility on ferroelectric properties of barium titanate ceramics. J. Am. Ceram. Soc. 42, 465-470 (1956).

37. Devries, R. C. \& Burke, J. E. Microstructure of barium titanate ceramics. J. Am. Ceram. Soc. 40, 200-206 (1957). 
38. Chou, J. F., Lin, M. H. \& Lu, H. Y. Ferroelectric domains in pressureless-sintered barium titanate. Acta Mater. 48, 3569-3579 (2000).

39. Cao, W. W. \& Randall, C. A. Grain size and domain size relations in bulk ceramic ferroelectric materials. J. Phys. Chem. Solids 57, 1499-1505 (1996).

40. Eriksson, M. et al. Ferroelectric domain structures and electrical properties of finegrained lead-free sodium potassium niobate ceramics. J. Am. Ceram. Soc. 94, 3391-3396 (2011).

41. Scott, J. F. Cylinder stress in nanostructures: effect on domains in nanowires, nanotubes, and nano-disks. J. Phys.: Condens. Matter 26, 212202 (2014).

42. Li, S. P., Cao, W. W. \& Cross, L. E. The extrinsic nature of nonlinear behavior observed in lead zirconate titanate ferroelectric ceramic. J. Appl. Phys. 69, 7219-7224 (1991)

43. Zhang, Q. M., Wang, H., Kim, N. \& Cross, L. E. Direct evaluation of domain-wall and intrinsic contributions to the dielectric and piezoelectric response and their temperature dependence on lead zirconate-titanate ceramics. J. Appl. Phys. 75, 454 (1991).

44. Demartin, M. \& Damjanovic, D. Dependence of the direct piezoelectric effect in coarse and fine grain barium titanate ceramics on dynamic and static pressure. Appl. Phys. Lett. 68, 3046-3048 (1996).

45. Damjanovic, D. Stress and frequency dependence of the direct piezoelectric effect in ferroelectric ceramics. J. Appl. Phys. 82, 1788-1797 (1997).

46. Damjanovic, D. Contributions to the piezoelectric effect in ferroelectric single crystals and ceramics. J. Am. Ceram. Soc. 88, 2663-2676 (2005).

47. Arlt, G. \& Pertsev, N. A. Force constant and effective mass of $90^{\circ}$ domain walls in ferroelectric ceramics. J. Appl. Phys. 70, 2283-2289 (1991).

48. Luchaninov, A. G., Shil'nikov, A. V., Shuvalov, L. A. and Shipkova, I. Ju. The domain processes and piezoeffect in polycrystalline ferroelectrics. Ferroelectrics 98, 123-126 (1989).

49. Viola, G., Chong, K. B., Guiu, F. \& Reece, M. J. Role of internal field and exhaustion in ferroelectric switching. J. Appl. Phys. 115, 034106 (2014)

50. Cao, W. Ferroelectrics: The strain limits on switching. Nat. Mater. 4, 727-728 (2005).

51. Martirena, H. T. \& Burfoot, J. C. Grain-size effects on properties of some ferroelectric ceramics. J. Phys. C: Solid State Phys. 7, 3182-3192 (1974).

52. Waser, R. Solubility of hydrogen defects in doped and undoped $\mathrm{BaTiO}_{3}$. J. Am Ceram. Soc. 71, 58-63 (1988)

53. Yoo, I. K. \& Desu, S. B. Fatigue parameters of lead zirconate titanate thin films. Mater. Res. Soc. Symp. Proc. 243, 323 (1992).

54. Scott, J. F. \& Paz de Arajuo, C. A. Ferroelectric memories. Science 246, 1400-1405 (1989).

55. He, L. \& Vanderbilt, D. First-principles study of oxygen-vacancy pinning of domain walls in $\mathrm{PbTiO}_{3}$. Phys. Rev. B 68, 134103 (2003).

56. Choi, Y., Hoshina, T., Takeda, H. \& Tsurumi, T. Effect of oxygen vacancy and oxygen vacancy migration on dielectric response of $\mathrm{BaTiO}_{3}$-Based Ceramics. Jpn. J. Appl. Phys. 50, 031504 (2011).

57. Oyama, T., Wada, N. \& Takagi, H. Trapping of oxygen vacancy at grain boundary and its correlation with local atomic configuration and resultant excess energy in barium titanate: a systematic computational analysis. Phys. Rev. B 82, 134107 (2010).

58. Kang, B. S., Choi, S. K. \& Park, C. H. Diffuse dielectric anomaly in perovskite-type ferroelectric oxides in the temperature range of $400-700^{\circ}$ C. J. Appl. Phys. 94, 1904-1911 (2003).

59. Karthik, J., Damodaran, A. R. \& Martin, L. W. Effect of $90^{\circ}$ domain walls on the low-field permittivity of $\mathrm{PbZr}_{0.2} \mathrm{Ti}_{0.8} \mathrm{O}_{3}$ thin films. Phys. Rev. Lett. 108, 167601 (2012).

60. Yan, H. X. et al. The contribution of electrical conductivity, dielectric permittivity and domain switching in ferroelectric hysteresis loops. J. Adv. Dielectr. 1, 107-118 (2011).

61. Viola, G. et al. Contribution of piezoelectric effect, electrostriction and ferroelectric/ferroelastic switching to strain-electric field response of dielectrics. J. Adv. Dielectr. 3, 1350007 (2013).

\section{Acknowledgments}

This work was financially supported by the Specialized Research Fund for the Doctoral Program of Higher Education (Grant No. 20130131110006) and the National Natural Science Foundation of China (Grant No. 51172128). The authors thank Prof. D. R. Chen of Shandong University for providing the hydrothermally-synthesized fine $\mathrm{BaTiO}_{3}$ powder. We would also like to thank China Scholarship Council for the financial support of 1 years research in the UK. One of the authors, V. Koval, acknowledges the support of the Grant Agency of Slovak Academy of Sciences through Grant No. 2/0057/14

\section{Author contributions}

T.Y.Q., Z.J.L., K.V., W.C.L., R.M., V.G. and Y.H.X. wrote the main manuscript text, W.Y.Q prepared Figure 1, T.Y.Q. prepared Figures 2-6 and Figures S1, 3, S.B.G. and Y.H.T prepared Figures S2. All authors reviewed the manuscript.

\section{Additional information}

Supplementary information accompanies this paper at http://www.nature.com/ scientificreports

Competing financial interests: The authors declare no competing financial interests.

How to cite this article: Tan, Y. et al. Unfolding grain size effects in barium titanate ferroelectric ceramics. Sci. Rep. 5, 9953; DOI:10.1038/srep09953 (2015).

This work is licensed under a Creative Commons Attribution 4.0 International License. The images or other third party material in this article are included in the article's Creative Commons license, unless indicated otherwise in the credit line; if the material is not included under the Creative Commons license, users will need to obtain permission from the license holder in order to reproduce the material. To view a copy of this license, visit http://creativecommons.org/licenses/by/4.0/ 